\title{
A PROPOSAL FOR AN X-RAY EXPERIMENT FOR COS-B
}

\author{
A. P. WILLMORE
}

Mullard Space Science Laboratory, Physics Dept., University College London, London, England

\section{Introduction}

It was proposed that the COS payload should include an experiment to study cosmic $\gamma$-rays and one for cosmic X-rays. From a scientific point of view the association of these experiments is clearly valuable, the more so in view of Ogelmann's suggestion that the $\gamma$-rays observed by Clark, Garmire and Kranshaar and by Hutchinson represent the high energy extension of the spectra of galactic X-ray sources. It may, however, be argued that the X-ray sources can independently be studied and that, in any case, the most important sources near the galactic plane will be well enough known by the launch date of the COS satellite. This argument may not be valid in view of the observed variability of X-ray sources, but in any case a strong case can be made for the proposed kind of experiment.

The suggested objectives of the experiment, more or less in descending order of weight as far as this design is concerned, would be (a) to measure with very high precision the location of discrete sources, to assist in optical identifications; (b) to improve knowledge of their spectral characteristics; (c) to study short period variations, and (d) to discover new sources.

Only a few of the known sources can be identified with any confidence with optically known objects - in this respect X-ray astronomy presents a close parallel with the early days of radio astronomy. The reason is simply that existing techniques do not in general permit the location of the source to better than about $1^{\circ}$, leaving an area of uncertainty containing many thousands of stars. No experiment is at present planned anywhere on the time scale we are considering which will enable sources to be located with the precision necessary to give only 1-10 known stars (i.e. down to say $20^{\mathrm{m}}$ ) in the field of view. The precision required for this is about 10 arc sec in the worst case. The planned U.S. experiments will probably not achieve better than an arc minute, at which level the ambiguity will still include up to a hundred stars.

An experiment with a rather large field of view (perhaps $45^{\circ}$ included angle) and capable of discriminating all the sources in this field, is also valuable for spectral studies and for studies of variability. The planned experiments on SAS-A and SAS-C (with one exception) are not of this character, except for strong sources, because of their limited throughput. Thus, the inclusion of a suitable wide-field experiment on COS-B would give considerable new information on the spectral characteristics of fainter sources, including probably extra-galactic sources, only one of which (M-87) is known at present. Finally, by covering the same region of the sky for long periods - 10-20 days - the short period variability of many sources will be observed, including both flaring of the type exhibited by SCO-X1, if this occurs in other sources, and 
possibly also emission from flare stars which has not so far been observed at all in $\mathrm{X}$-rays. The proposed experiment has an extremely high sensitivity, the collecting efficiency being 10 and 100 times greater than the two low angular resolution experiments on SAS-A and when combined with the long pointing time this should enable new sources, many of which are likely to be extra-galactic, to be discovered.

\section{Experimental Method}

The proposal for COS-A, made by Dr. Pounds, included a novel device in the form of a moving collimator. The effective application of this system required a spacecraft with 3-axis stabilisation and low rates of angular drift. In the case of COS-B which is to be spin-stabilised this system can no longer be used. However, Pound's device is an example of a general class of such collimators which the writer has recently studied theoretically. An alternative form, which is not in any respect inferior to the moving collimator and which has certain advantages, is a rotation collimator. In this case the two grids are maintained at a fixed spacing, and rotated about their common axis. The X-rays from a source off this axis are modulated at a frequency depending on their position. This system is compatible with the proposed COS-B, the spacecraft providing the rotation so that the collimator remains fixed relative to the spacecraft. For both the versions of the experiment, the rate of drift of the pointing direction is the main limiting factor in the determination of source position. The rotating satellite has the advantages that the gyroscopic rigidity of the satellite automatically minimises the steady drift, and that the nutation can readily be damped by conventional means. On the other hand, the spin rate will then be higher than the period associated with the moving collimator, so that the modulating frequencies will be higher in this case and cannot directly be telemetered. Since the modulating waveform has the fundamental period of the spin frequency, the counts can be accumulated in a buffer store, the addresses in the store being determined by a sector generator synchronised to the sun, if sufficient accuracy can be obtained, or to a star. The weight and power figures given assume that the core store and sector generator are included in the experiment although they are also required for the $\gamma$-ray experiment and it would obviously be more efficient to combine them. In addition, because of the high stability required, it is assumed that an independent sub-structure is provided for the X-ray experiment and quasi-kinematically attached to the spacecraft.

A detailed description of the experiment is not given, because it is so similar to that proposed by Dr. Pounds. In particular, the use of a photomultiplier to determine the orientation of the collimator is included. However, some comments and explanations may be useful. The orbit selected for COS-B is advantageous for the X-ray experiment also, because the observing time is influenced by similar factors to the $\gamma$-ray experiment. As for that experiment, advantage has been taken of this to reduce the size of the detectors without reducing the sensitivity. This factor, together with the elimination of the high precision drive for the collimators reduces the weight requirement. It has been assumed that the experiment would be located in the annular space 
around the central tube, though a structural re-arrangement might possibly be more sensible. The limiting factors for the angular accuracy of the experiment are the drift rate of the spin axis and the telemetry rate (assuming the requirements for nutation are satisfied). The companion visible star system enables the position of the axis to be determined in about 1 minute of time. A certain quantity of data is thus collected. If sufficient telemetry is available for this to be transmitted every minute, then the effective image degradation is the spin axis movement in $1 \mathrm{~min}$. At a lower telemetry rate, the position is determined less often (the quantity of data to be transmitted increased only quite slowly with the period) and the image degradation is worse. The design presented assumes the figures of the COS-B feasibility study.

A total length available of $70 \mathrm{~cm}$ has been assumed, though an extension to the full length of the spacecraft would be desirable and would result in a useful improvement in angular accuracy.

\section{Experiment parameters}

\section{Collimator and detector package}

Size: Approximately a cylinder $35 \mathrm{~cm}$ diameter, $70 \mathrm{~cm}$ long, constrained as necessary to fit in one of the large equipment compartments.

Detector diameter (active area) $30 \mathrm{~cm}$.

Weight $18.5 \mathrm{~kg}$.

Electronics package (not including cabling) $6 \mathrm{~kg}$.

Total weight $24.5 \mathrm{~kg}$.

Mounting requirements: To be aligned with an accuracy of 0.5 to the spacecraft spin axis.

Stabilisation: The spacecraft should be spin-stabilised, with a drift rate of less than $0.5 /$ orbit and an amplitude of nutation not exceeding $5 "$ arc. (This may necessitate a fluid rather than a ball-in-tube nutation damper). A spin rate of 10 r.p.m. is suitable.

Spin timing pulse: A timing pulse is required, once each rotation relative to fixed stars. Over a five minute period, no individual pulse should depart from the position corresponding to an exact rotation reference by more than $15 \mathrm{arcsec}$. On the other hand, neither the actual position of this pulse in rotation nor its long-term stability are of any importance.

Telemetry: The most efficient method of interfacing the experiment with the telemetry via a core store requires a detailed study from a system point of view, and this has not been possible in the time available for producing this proposal. A method has been worked out which may not be an optimum but which would work.

Each spin cycle would be divided by a sector generator synchronised to the spin timing pulse into $8 \mathrm{~K}$ segments. The counter output would be divided by pulse height analysis into two energy ranges, the counts from each in each sector being stored in a 4 bit count. In addition an 8-bit count would be required for the star tracker. Thus, the total memory storage required is $16 \mathrm{~K}$ of 8 -bit words, half word access being required to half the store. This store would be read out via the telemetry on a continuous cycle, the read-out period being $1280 \mathrm{sec}$ at a bit rate of $100 \mathrm{~b} / \mathrm{s}$. Together 
with $100 \mathrm{~b} / \mathrm{s}$ this gives the $200 \mathrm{~b} / \mathrm{s}$ capacity envisaged in the design study, but a small increase would of course be necessary to satisfy the requirements of the spacecraft housekeeping.

Power: A reasonable power allocation for the experiment would be 4 watts.

Angular accuracy of position measurement: The effective image size of the collimator depends on the characteristics of the collimator, together with blurring resulting from the instability of the spin direction:

$\begin{array}{ll}\text { Image size due to collimator } & 90 \text { arc sec. } \\ \text { drift in core recycling period } & 20 \\ \text { nutation (total amplitude) } & \frac{10}{120} \text { arc sec. }\end{array}$

For faint sources which are only just detected this would be the error in position measurement. For bright sources, of which there would be many because of the high sensitivity of the experiment, the position error would be about $\frac{1}{10}$ of the image size, or 12 arc sec, together with an error of about 9 arc sec contributed by the star tracker. Combining these errors quadratically since they are independent we obtain an overall error of 15 arc sec.

Ambiguity in position: The rotation collimator determines two position coordinates for the sources in the view field (in this respect it differs from the original proposal of Dr. Pounds; the difference arises essentially because both frequency and phase information in the modulation are available) but because of its two-fold symmetry about the rotation axis there is a corresponding ambiguity in the azimuth angle of the sources To resolve this ambiguity, two sets of measurements are required. The simplest scheme appears to be to displace either the spacecraft spin axis, or the axis of the experiment, periodically through a small angle. An angle larger than the image size of the experiment, but smaller than is detectable by the $\gamma$-ray experiment seems a good choice; we propose $5 \mathrm{arc} \min$. Thus, one orbit might be spent on one position, followed by a displacement for the succeeding orbit. If, however, a careful study shows that the spin axis drift is sure under all circumstances to lie between 0.1 and 0.5 degrees per orbit, this provision would be unnecessary. 